\title{
Channel Allocation for UMTS Multimedia Broadcasting and Multicasting
}

\author{
Yen-Cheng Lai, Student Member, IEEE, Phone Lin, Senior Member, IEEE, Yuguang Fang, Fellow, IEEE, \\ and Wei-Hao Chen
}

\begin{abstract}
GPP 23.246 proposed the Multimedia Broadcast Multicast Service (MBMS) to deliver multicasting content in the Universal Mobile Telecommunications System (UMTS). In MBMS, the common logical channel is enabled to serve multiple MBMS calls at the same time. Use of the common logical channel may cause interference to the dedicated logical channels serving the traditional calls. To more efficiently utilize the radio resource to serve both traditional and MBMS calls, this paper proposes two channel allocation algorithms: Reserved Resource for Multicasting (RRM) and Unreserved Resource for Multicasting (URM). We propose analytic models and conduct simulation experiments to investigate customer Satisfaction Indication $(S I)$ for the two algorithms. Our study indicates that URM outperforms RRM in terms of customer $S I$.
\end{abstract}

Index Terms-Channel allocation, multimedia broadcast multicast service (MBMS), performance modeling, soft handoff, universal mobile telecommunications system (UMTS).

\section{INTRODUCTION}

$\mathbf{T}$ HE 3GPP working group proposed the Multimedia Broadcast Multicast Service (MBMS) for multicasting multimedia content in the Universal Mobile Telecommunications System (UMTS) [8]. In UMTS, the Wideband Code Division Multiple Access (WCDMA) is the air interface between the User Equipments (UEs) and the UMTS Terrestrial Radio Access Network (UTRAN). Two wireless transmission modes for MBMS are defined in WCDMA, which are Point-ToPoint (PTP) and Point-To-Multipoint (PTM). In PTP and PTM modes, the dedicated logical channels and the common logical

Manuscript received June 16, 2007; revised November 11, 2007; accepted December 19, 2007. The associate editor coordinating the review of this paper and approving it for publication was M. Guizani. Y.-C. Lai's work was partially supported by the National Science Council (NSC), R.O.C., under Contract NSC-97-2218-E-002-026. P. Lin's work was partially supported by the National Science Council (NSC), R.O.C., under the contract number NSC-96-2627-E-002-001-, NSC-96-2811-E-002-010, NSC-96-2628E-002-002-MY2 and NSC-95-2221-E-002-091-MY3, Ministry of Economic Affairs (MOEA), R.O.C., under contract number 93-EC-17-A-05-S1-0017, Telcordia Applied Research Center, Taiwan Network Information Center (TWNIC), and Excellent Research Projects of National Taiwan University, 97R0062-05. Y. Fang's work was partially supported by US National Science Foundation under grant CNS-0721744 and the National Science Council (NSC), R.O.C., under the NSC Visiting Professorship with contract number NSC-96-2811-E-002-010.

Y.-C. Lai is with the Department of Computer Science \& Information Engineering, National Taiwan University, Taipei 106, Taiwan, R.O.C. (e-mail: yclai@csie.ntu.edu.tw).

P. Lin is with the Department of Computer Science \& Information Engineering and the Graduate Institute of Networking \& Multimedia, National Taiwan University, Taipei 106, Taiwan, R.O.C. (e-mail: plin@ csie.ntu.edu.tw).

Y. Fang is with the Department of Electrical \& Computer Engineering, University of Florida, USA (e-mail: fang@ece.ufl.edu).

W.-H. Chen is with the Mobile Application Software Division, High Tech Computer Corp., Taipei 231, Taiwan, R.O.C. (e-mail: Albert_Chen@htc.com.tw).

Digital Object Identifier 10.1109/T-WC.2008.070653 channels are used to deliver MBMS data to UEs, respectively. The MBMS users may receive MBMS data via a dedicated logical channel (by executing the radio bearer setup procedure [2]) or via a common logical channel (by simply tuning to the common logical channel without Node B's awareness). A common logical channel can serve more than one MBMS call at the same time. The drawback for the usage of a common logical channel is that there is no associated control channel for common logical channels to report the channel quality, and the power control can not be done. To cover the whole cell, the transmission power of a common logical channel should be large enough. As mentioned in [8], [15], in WCDMA, the power control brings significant capacity gain. The usage of a common logical channel may bring high interference to the dedicated logical channels, and the capacity for dedicated logical channels decreases. It is recommended to use a common logical channel to multicast the MBMS data while the number of MBMS calls is sufficiently large. 3GPP 25.346 proposed the Channel Type Switching procedure to switch an MBMS user between PTP and PTM modes for more flexible channel allocation, whose details can be found in [1].

In UMTS, the radio resources are not only used to serve the MBMS calls but also other circuit-switched/packet-switched calls (also known as traditional calls) where dedicated logical channels are used to deliver the user data. The radio resource allocation for both the MBMS and traditional calls is a critical issue, which affects QoS of both MBMS and traditional calls significantly. This paper proposes two channel allocation algorithms: Reserved Resource for Multicasting (RRM) and Unreserved Resource for Multicasting (URM). We propose analytic models and conduct simulation experiments to investigate the performance of the two algorithms.

\section{Channel Allocation Algorithms}

This section describes the RRM and URM algorithms. Suppose that a UE can only carry a traditional call or an MBMS call at a time. A traditional call is served by the dedicated logical channel. An MBMS call is served by either the dedicated logical channel (i.e., PTP mode) or the common logical channel (i.e., PTM mode). As mentioned in [1], [3], [14], the dedicated logical channel supports soft handoff, and the common logical channel supports soft combining, where a handoff ${ }^{1}$ call can use different logical channels to connect to more than one Node B at the same time in the overlapping

\footnotetext{
${ }^{1}$ The handoff process is that when a communicating UE moves from one cell to another, the channel in the old Node B is released, and a channel is required in the new Node $B$.
} 
area between adjacent cells. The details of soft handoff model will be elaborated in Section III.

Assume that there are $C$ logical channels in a cell. A Node B turns on at most one common logical channel to serve the MBMS calls. We assume that turning on one common logical channel causes damage to $\theta$ dedicated logical channels. The $\theta$ value depends on several physical layer factors (e.g., path loss). The analysis of $\theta$ is out of the scope of this paper, and readers may refer to [5], [6].

Suppose that the system is at one of the two states, $\mathbf{O N}$ and OFF. At the ON state, the common logical channel is turned on to serve more than one MBMS user simultaneously, and there are at most $C^{*}=C-\theta-1$ dedicated logical channels. At the OFF state, the common logical channel is turned off, and there are $C$ dedicated logical channels. A FIFO handoff queue is executed in each cell to buffer handoff calls that cannot be served immediately due to the lack of channels in the new cell. The RRM and URM algorithms are exercised as follows:

Algorithm RRM. The RRM reserves a common logical channel to serve the new or handoff MBMS calls. The state is set to $\mathbf{O N}$. For a new or handoff traditional call arrival, the system check whether there are free dedicated logical channels. If so, one dedicated logical channel is allocated to serve the request. Otherwise, the new traditional call request is blocked, and the handoff traditional call is buffered in the handoff queue. If the traditional handoff call moves out of the overlapping area before a dedicated logical channel is available, the call is forced to terminate.

When a traditional call is completed, RRM releases one dedicated logical channel. Then the system checks whether there are handoff traditional calls buffered in the handoff queue. If so, the released channel is assigned to a handoff call in the queue.

Algorithm URM. The URM does not reserve any common logical channel to serve the MBMS calls in advance. For a new or handoff traditional call request, URM takes the same actions as that in RRM. For an MBMS call request, the system decides whether the dedicated logical channel or the common logical channel can be used to serve the MBMS call. If number of MBMS calls in the system is larger than $\theta$, then URM turns on the common logical channel for the incoming MBMS call. We consider the following two cases to handle a new or handoff MBMS call request.

Case URM1: The system is at the $\mathbf{O N}$ state. The request is served by the common logical channel.

Case URM2: The system is at the OFF state. Let $n_{t}$ be the number of traditional call users in the system. If $C-n_{t} \leq \theta$, the common logical channel is not turned on. The system determines whether there are free dedicated logical channels. If so, one dedicated logical channel is allocated to serve the new or handoff MBMS call request. Otherwise, the new MBMS call request is blocked and the handoff MBMS call request is buffered in the handoff queue.

If $C-n_{t}>\theta$, URM checks the number of MBMS calls in the system. If the number of MBMS calls $<\theta$, one dedicated logical channel is allocated to serve the new or handoff MBMS call request. Otherwise (i.e., the number of MBMS calls $\geq \theta$ ), the system turns on the common logical channel to serve the MBMS request, and URM switches the state from OFF to ON. The MBMS calls (that are currently served by the dedicated logical channels) are moved to the common logical channel. The new or handoff MBMS call is then served by the common logical channel.

When a served MBMS call leaves the system at the OFF state, one dedicated logical channel is released. The URM checks whether there are handoff calls in the handoff queue. If so, the released channel is assigned to one buffered handoff call. On the other hand, if the system is at the ON state, URM checks whether the number of MBMS calls in the system is less than or equal to $\theta$. If so, to save the bandwidth, it is preferred to use the dedicated logical channels (i.e., PTP mode) to serve the MBMS calls. The following instructions are executed: First, URM moves the MBMS calls (that are currently served by the common logical channel) to the dedicated logical channels. The common logical channel is turned off, and the state switches from ON to OFF. Next, URM checks whether there are handoff calls in the handoff queue. If so, the released channel is assigned to one buffered handoff call.

\section{AnAlytic Models}

This section proposes analytical models for the RRM and URM algorithms. We consider a homogeneous system, where all cells are assumed to be identical. A handoff call can connect up to 2 radio links in the overlapping area between two adjacent cells. The traditional and MBMS call arrivals to a cell form Poisson processes with arrival rate $\lambda_{o, t}$ and $\lambda_{o, m}$, respectively. The call holding time $t_{c, t}\left(t_{c, m}\right)$ for a traditional call (an MBMS call) is exponentially distributed with the mean $1 / \mu_{t}\left(1 / \mu_{m}\right)$. The UE residence time $t_{i}$ in Cell $i$ has an exponential distribution with the density function $f_{m}\left(t_{i}\right)=\omega e^{-\omega t_{i}}$. This assumption will be relaxed by using general residence time distribution in our simulation experiments. The output measures derived in our models include:

- $\lambda_{h, t}\left(\lambda_{h, m}\right)$ : traditional (MBMS) handoff call arrival rate

- $P_{o, t}\left(P_{o, m}\right)$ : new call blocking probability for a traditional call (an MBMS call)

- $P_{f, t}\left(P_{f, m}\right)$ : the forced termination probability for a traditional handoff call (an MBMS handoff call), i.e., the probability that a traditional handoff call (an MBMS handoff call) is dropped because there is no free channel in the new cell

- $P_{n c, t}\left(P_{n c, m}\right)$ : the call incompletion probability for a traditional call (an MBMS call)

\section{A. Analysis for Algorithm RRM}

The RRM algorithm turns on the common logical channel to serve MBMS calls. The system is always at the $\mathbf{O N}$ state and the MBMS call requests can always be served. There are $C^{*}=C-\theta-1$ dedicated logical channels that can be used 


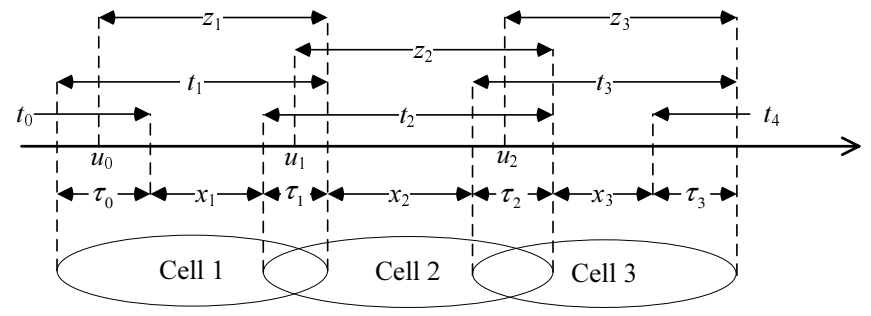

Fig. 1. The timing diagram for the soft handoff model

to serve the traditional calls. The MBMS call requests can be served in the system, and we have

$$
P_{o, m}=P_{f, m}=P_{n c, m}=0 .
$$

The soft handoff model in [14] is applied to derive the output measures for the traditional calls. Fig. 1 illustrates the timing diagram for the soft handoff model, where $t_{i}$ represents the time period during which a UE can receive the signal from Cell $i$.

Because the cells may overlap, the UE will enter overlapping area $i$ before it moves from Cell $i$ to Cell $i+1$. Let $x_{i}$ be the time period when the UE stays in the non-overlapping area of Cell $i$. Suppose that the overlapping period between Cell $i$ and Cell $i+1$ is $\tau_{i}$. Then $t_{i}$ can be expressed as

$$
t_{i}=\tau_{i-1}+x_{i}+\tau_{i} .
$$

A connecting traditional call utilizes one dedicated logical channel during the non-overlapping period $x_{i}$, and is looking for a second dedicated logical channel from Cell $i+1$ during $\tau_{i}$. Assume that the second dedicated logical channel is found at time $u_{i}$, and $z_{i}$ is the residual cell residence time in cell $i$. Then the channel occupancy time of the connecting traditional call in Cell $i+1$ is $\min \left(z_{i+1}\right.$, the remaining call holding time). Since cell residence time $t_{i}$ is exponentially distributed, from the memoryless property, $z_{i}$ also has the same distribution as $t_{i}$. Let $E\left[z_{i}\right]=1 / \omega, E\left[x_{i}\right]=1 / \eta$ and $E\left[\tau_{i}\right]=1 / \gamma$. Then from (1), we have

$$
E\left[z_{i}\right]=E\left[t_{i}\right]=E\left[\tau_{i-1}\right]+E\left[x_{i}\right]+E\left[\tau_{i}\right]
$$

or

$$
\frac{1}{\omega}=\frac{1}{\gamma}+\frac{1}{\eta}+\frac{1}{\gamma}=\frac{\gamma+2 \eta}{\gamma \eta} .
$$

From [7], [14], $\lambda_{h, t}$ and $P_{n c, t}$ for a traditional call can be computed as follows:

$$
\begin{aligned}
\lambda_{h, t} & =\frac{\eta \gamma}{\eta \gamma+(\eta+\gamma) \mu_{t}}\left[\lambda_{o, t}\left(1-P_{o, t}\right)+\lambda_{h, t}\left(1-P_{f, t}\right)\right] \\
& =\frac{\eta \gamma\left(1-P_{o, t}\right) \lambda_{o, t}}{(\eta+\gamma) \mu_{t}+\eta \gamma P_{f, t}}
\end{aligned}
$$

and

$$
P_{n c, t}=P_{o, t}+\frac{\eta \gamma\left(1-P_{o, t}\right) P_{f, t}}{(\eta+\gamma) \mu_{t}+\eta \gamma P_{f, t}} .
$$

To compute $P_{o, t}$ and $P_{f, t}$, we use a Markov process with states $\mathbf{s}(n)$, where $n \geq 0$ represents the number of traditional calls in a cell. Let $\Lambda_{t}=\lambda_{o, t}+\lambda_{h, t}$ be the net traditional call arrival rate (including new and handoff traditional calls) to a cell. Let $M_{t}=\mu_{t}+\omega$. Then $1 / M_{t}$ is the mean

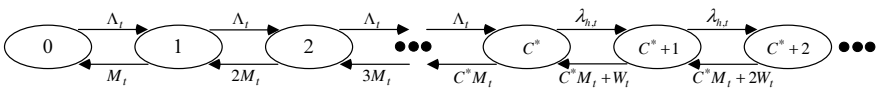

Fig. 2. The Markov process for RRM

channel occupancy time of a traditional call at a cell. Let $W_{t}=\mu_{t}+\gamma$. The $1 / W_{t}$ is the mean waiting time of an outstanding traditional handoff call (which is waiting for a second link). Fig. 2 illustrates the Markov process for RRM.

Let $\pi_{i}$ be the steady-state probability for $\mathbf{s}(n)$. From [14], the $\pi_{i}$ probability can be computed by

$$
\pi_{i}= \begin{cases}\frac{\Lambda_{t}^{i}}{i ! M_{t}^{i} \pi_{0},} & \text { if } i \leq C^{*} \\ \frac{\Lambda_{t}^{C^{*}} \lambda_{h, t}^{i-C^{*}}}{C^{*} ! M_{t}^{C^{*}} \prod_{1 \leq j \leq i-C^{*}}\left(C^{*} M_{t}+j W_{t}\right)} \pi_{0}, & \text { if } i>C^{*}\end{cases}
$$

Since $\pi_{0}+\pi_{1}+\cdots+\pi_{n}+\cdots=1$, we have

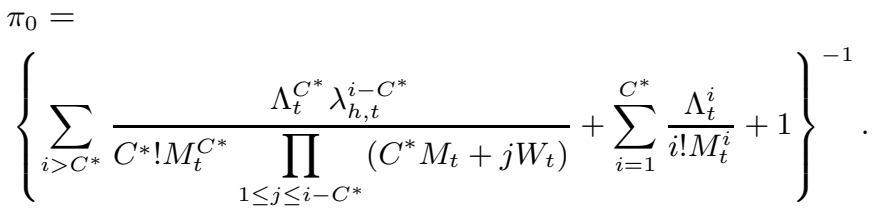

A new traditional call is blocked when the system is at state $\mathbf{s}(n)$ (where $n \geq C^{*}$ ) as it arrives. The call blocking probability for original traditional calls is

$$
P_{o, t}=\sum_{i \geq C^{*}} \pi_{i}
$$

$P_{f, t}$ is derived as follows. Suppose that a traditional handoff call $H_{t, n}$ enters a new cell (i.e., the overlapping area) at time $t$, and the cell is at state $\mathbf{s}(n)$ (where $n=C^{*}+j$ and $j \geq 0$ ). In this situation, all dedicated logical channels in the new cell are busy, and $j$ outstanding traditional handoff calls arrive at the cell earlier than $H_{t, n}$. The $H_{t, n}$ call request is buffered in the handoff queue in the new cell. Let $G_{n}^{(k)}$ be the probability that $k$ outstanding traditional handoff calls leave the new cell due to completion or dropping before $H_{t, n}$ leaves the cell. Suppose that among the $C^{*}+j$ traditional calls (including $C^{*}$ traditional calls which are being served, and $j$ outstanding traditional handoff calls), the first outstanding traditional call leaves the handoff queue (either completes, expires, or leaves the cell) at time $t+t_{1}$. Under the memoryless property of the exponential distribution, the probability density function for $t_{1}$ is given by

$$
f_{n}^{(1)}\left(t_{1}\right)=\left(C^{*} M_{t}+j W_{t}\right) e^{-\left(C^{*} M_{t}+j W_{t}\right) t_{1}} .
$$

Let $t_{w}$ be the maximum waiting time of an outstanding traditional handoff call (which is waiting for a second link). Then we have $t_{w}=\min$ (remaining call holding time, overlapping period). By applying the memoryless property of the exponential distribution, the density function $f_{w}$ for $t_{w}$ is

$$
f_{w}\left(t_{w}\right)=W_{t} e^{-W_{t} t_{w}} .
$$


Then we have the probability $G_{n}^{(1)}$ (that $H_{t, n}$ keeps searching the second link within the time period $t_{w}$ ) as

$$
\begin{aligned}
G_{n}^{(1)} & =\operatorname{Pr}\left\{t_{w}>t_{1}\right\}=\int_{t_{w}=0}^{\infty} \int_{t_{1}=0}^{t_{w}} f_{w}\left(t_{w}\right) f_{n}^{(1)}\left(t_{1}\right) d t_{1} d t_{w} \\
& =\frac{C^{*} M_{t}+j W_{t}}{C^{*} M_{t}+(j+1) W_{t}} .
\end{aligned}
$$

With the memoryless property, $G_{n}^{(k)}$ for $n \geq C^{*}$ can be computed recursively by using

$$
G_{n}^{(k)}=G_{n}^{(1)} \times G_{n-1}^{(k-1)} .
$$

The probability that a traditional handoff call (entering the new cell at state $\mathbf{s}(n)$ ) can get a free channel is given by

$$
\operatorname{Pr}\{\text { get a channel } \mid \mathbf{s}(n)\}=G_{n}^{\left(n-C^{*}+1\right)}, \quad \text { for } n \geq C^{*}
$$

where $G_{n}^{\left(n-C^{*}+1\right)}$ is obtained by using (6). From (7), we have the probability that a traditional handoff call (entering the new cell at state $\mathbf{s}(n))$ is forced to terminate as

$$
\operatorname{Pr}\{\text { force termination } \mid \mathbf{s}(n)\}=1-G_{n}^{\left(n-C^{*}+1\right)}, \quad \text { for } n \geq C^{*},
$$

and the force termination probability $P_{f, t}$ is

$$
P_{f, t}=\sum_{n \geq C^{*}}^{\infty}\left(1-G_{n}^{\left(n-C^{*}+1\right)}\right) \pi_{n} .
$$

With (2)-(4) and (8), the following iterative approach is executed to compute $\lambda_{h, t}, P_{o, t}, P_{f, t}$, and $P_{n c, t}$ :

Step 1. Select an initial value for $\lambda_{h, t}$.

Step 2. $\lambda_{h, t, \text { old }} \leftarrow \lambda_{h, t}$.

Step 3. Compute $P_{o, t}$ and $P_{f, t}$ by using (4) and (8), respectively.

Step 4. Compute $\lambda_{h, t}$ by using (2).

Step 5. If $\left|\lambda_{h, t}-\lambda_{h, t, \text { old }}\right|>\delta \lambda_{h, t}$, then go to Step 2 . Otherwise, go to Step 6 . Note that $\delta$ is a predefined threshold set to $10^{-7}$.

Step 6. The values for $\lambda_{h, t}, P_{o, t}$, and $P_{f, t}$ converge. Compute $P_{n c, t}$ by using (3).

\section{B. Analysis for Algorithm URM}

This section proposes an analytic model for the URM algorithm. We model the algorithm by using a three-dimensional Markov process. The state $\mathbf{s}(i, j, k)$ denotes the state where a cell is occupied by $i$ MBMS calls, $j$ traditional calls (which are being served), and $k$ outstanding traditional handoff calls (which are waiting for the second link), respectively. Then the state space $\mathbf{S}$ for this Markov process of URM is

$$
\mathbf{S}=\{\mathbf{s}(i, j, k) \mid i \geq 0,0 \leq j \leq C, k \geq 0\} .
$$

Let $\pi_{i, j, k}$ be the steady-state probability for state $\mathbf{s}(i, j, k)$. By convention, $\pi_{i, j, k}=0$ if state $\mathbf{s}(i, j, k) \notin \mathbf{S}$. For all reachable sates $\mathbf{s}(i, j, k) \in \mathbf{S}$, we have

$$
\sum_{\mathbf{S}(i, j, k) \in \mathbf{S}} \pi_{i, j, k}=1 .
$$

Fig. 3 illustrates the state transition diagram for URM, where the soft handoff model is applied to both traditional and MBMS calls. Due to page limitation, the description for the

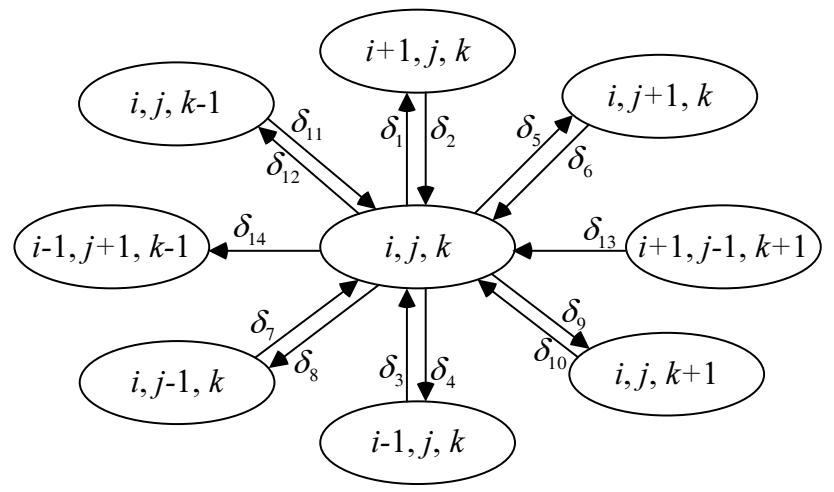

Fig. 3. The Markov process for URM

state transitions is omitted, which can be found in pp. 1-3 [10]. The balance equation for the Markov process is expressed as

$$
\begin{aligned}
& \left(\delta_{1}+\delta_{4}+\delta_{5}+\delta_{8}+\delta_{9}+\delta_{12}+\delta_{14}\right) \pi_{i, j, k} \\
= & \delta_{1} \pi_{i+1, j, k}+\delta_{3} \pi_{i-1, j, k}+\delta_{6} \pi_{i, j+1, k}+\delta_{7} \pi_{i, j-1, k} \\
+ & \delta_{10} \pi_{i, j, k+1}+\delta_{11} \pi_{i, j, k-1}+\delta_{13} \pi_{i+1, j-1, k+1} .
\end{aligned}
$$

From (9) and (10), the steady state probability $\pi_{i, j, k}$ can be solved by using the successive over-relaxation method [4]. The $P_{o, t}, P_{f, t}, P_{o, m}$, and $P_{f, m}$ for Algorithm URM can be derived as follows.

In URM, a new traditional call is blocked when the system is at the state $\mathbf{s}(i, j, k)$ where $0 \leq i \leq \theta, C-i \leq j \leq C$, $k \geq 0$ or $i \geq \theta+1, C^{*} \leq j \leq C, k \geq 0$, and we have

$P_{o, t}=\sum_{i=0}^{\theta} \sum_{j=C-i}^{C} \sum_{k \geq 0} \pi_{i, j, k}+\sum_{i \geq \theta+1} \sum_{j=C^{*}}^{C} \sum_{k \geq 0} \pi_{i, j, k}$.

The derivation of $P_{f, t}$ for URM is similar to that of $P_{f, t}$ for RRM. Due to page limitation, the details are described in pp. 4-8 [10], and $P_{f, t}$ is given by

$$
\begin{aligned}
P_{f, t} & =\sum_{i=0}^{\theta} \sum_{j=C-i}^{C} \sum_{k \geq 0}\left(1-G_{i, j, k}^{(k+i-C+j+1)}\right) \pi_{i, j, k} \\
& +\sum_{i \geq \theta+1} \sum_{j=C^{*}+1}^{C} \sum_{k \geq 0}\left(1-G_{i, j, k}^{(k+i-C+j+1)}\right) \pi_{i, j, k} \\
& +\left.\sum_{i \geq \theta+1} \sum_{k \geq 0}\left(1-D_{i, j, k}^{(k+1)}\right) \pi_{i, j, k}\right|_{j=C^{*}} .
\end{aligned}
$$

In (11), $G_{i, j, k}^{(k+i-C+j+1)}$ can be obtained by iterating the following equations:

$$
G_{i, j, k}^{(l)}=\left\{\begin{aligned}
G_{i, j, k}^{(1)}, & \text { if } 0 \leq i \leq \theta, j=C-i, k=0 ; \\
G_{i, j, k}^{(1)} & \times\left(L_{i, j, k} G_{i-1, j, k}^{(l-1)}+H_{i, j, k} G_{i, j, k-1}^{(l-1)}+\right. \\
& \left.M_{i, j, k} G_{i, j-1, k}^{(l-1)}+N_{i, j, k} G_{i-1, j+1, k-1}^{(l-1)}\right), \\
& \text { if } 0 \leq i \leq \theta, j=C-i, k>0 \\
& \text { or } 1 \leq i \leq \theta, C-i<j \leq C, k \geq 0 \\
& \text { or } i \geq \theta+1, C^{*}+1<j \leq C, k \geq 0 ; \\
& \times\left(L_{i, j, k} G_{i-1, j, k}^{(l-1)}+H_{i, j, k} G_{i, j, k-1}^{(l-1)}+\right. \\
G_{i, j, k}^{(1)} & \left.M_{i, j, k}^{(l-i+C-j)}+N_{i, j, k}^{(l-1, k} G_{i-1, j+1, k-1}^{(l-1)}\right), \\
& \text { if } i \geq \theta+1, j=C^{*}+1, k \geq 0 .
\end{aligned}\right.
$$

where $G_{i, j, k}^{(1)}=\frac{(C-j) M_{m}+j M_{t}+(i-C+j) W_{m}+k W_{t}}{(C-j) M_{m}+j M_{t}+(i-C+j) W_{m}+(k+1) W_{t}}$ 
and

$$
\begin{aligned}
& (C-j) M_{m}+(i-C+j) W_{m} \\
& \overline{(C-j) M_{m}+j M_{t}+(i-C+j) W_{m}+k W_{t}}, \\
& \text { if } 1 \leq i \leq \theta, C-i<j \leq C, k=0 \\
& \text { or } i \geq \theta+1, C^{*}+1 \leq j \leq C, k=0 \text {; } \\
& \frac{[(i-C+j) /(k+i-C+j)](C-j) M_{m}}{(C-j) M_{m}+j M_{t}+(i-C+j) W_{m}+k W_{t}} \\
& +\frac{(i-C+j) W_{m}}{(C-j) M_{m}+j M_{t}+(i-C+j) W_{m}+k W_{t}}, \\
& L_{i, j, k}=\left\{\begin{array}{r}
(C-j) M_{m}+j M_{t}+(i-C+j) W_{m} \\
\text { if } 1 \leq i \leq \theta, C-i<j \leq C-1, k>0
\end{array}\right. \\
& \text { or } i \geq \theta+1, C^{*}+1 \leq j \leq C-1, k>0 \text {; } \\
& \frac{(i-C+j) M_{m}}{j M_{t}+(i-C+j) W_{m}+k W_{t}}, \\
& \text { if } 1 \leq i \leq \theta, j=C, k>0 \\
& \text { or } i \geq \theta+1, j=C, k>0 \text {; } \\
& 0 \text {, otherwise. } \\
& \frac{j M_{t}+k W_{t}}{(C-j) M_{m}+j M_{t}+k W_{t}}, \\
& \text { if } 1 \leq i \leq \theta, j=C-i, k>0 \text {; } \\
& {[k /(k+i-C+j)] j M_{t}+k W_{t}} \\
& \overline{(C-j) M_{m}+j M_{t}+(i-C+j) W_{m}+k W_{t}} \text {, } \\
& \text { if } 1 \leq i \leq \theta, C-i<j \leq C-1, k>0 \\
& \text { or } i \geq \theta+1, C^{*}+1 \leq j \leq C-1, k>0 \text {; } \\
& H_{i, j, k}= \\
& \frac{[k /(k+i-C+j)] j M_{t}+k W_{t}}{j M_{t}+(i-C+j) W_{m}+k W_{t}} \\
& \text { if } 1 \leq i \leq \theta, j=C, k>0 \\
& \text { or } i \geq \theta+1, j=C, k>0 \text {; } \\
& 1, \text { if } i=0, j=C, k>0 \text {; } \\
& 0 \text {, otherwise. }
\end{aligned}
$$$$
\frac{j M_{t}}{(C-j) M_{m}+j M_{t}+(i-C+j) W_{m}}
$$$$
\text { if } 1 \leq i \leq \theta, C-i<j \leq C, k=0
$$$$
\text { or } i \geq \theta+1, C^{*}+1 \leq j \leq C, k=0 \text {; }
$$$$
\frac{[(i-C+j) /(k+i-C+j)] j M_{t}}{(C-j) M_{m}+j M_{t}+(i-C+j) W_{m}+k W_{t}},
$$$$
M_{i, j, k}=
$$$$
\text { if } 1 \leq i \leq \theta, C-i<j \leq C-1, k>0
$$$$
\text { or } i \geq \theta+1, C^{*}+1 \leq j \leq C-1, k>0 \text {; }
$$$$
\frac{[(i-C+j) /(k+i-C+j)] j M_{t}}{j M_{t}+(i-C+j) W_{m}+k W_{t}},
$$$$
\text { if } 1 \leq i \leq \theta, j=C, k>0
$$$$
\text { or } i \geq \theta+1, j=C, k>0 \text {; }
$$

0 , otherwise

$$
N_{i, j, k}=\left\{\begin{array}{l}
\frac{(C-j) M_{m}}{(C-j) M_{m}+j M_{t}+k W_{t}}, \\
\text { if } 1 \leq i \leq \theta, j=C-i, k>0 \\
\frac{[k /(k+i-C+j)](C-j) M_{m}}{(C-j) M_{m}+j M_{t}+(i-C+j) W_{m}+k W_{t}}, \\
\text { if } 1 \leq i \leq \theta, C-i<j \leq C-1, k>0 \\
\text { or } i \geq \theta+1, C^{*}+1 \leq j \leq C-1, k>0 ; \\
0, \text { otherwise. }
\end{array}\right.
$$

$D_{i, j, k}^{(k+1)}$ in (11) can be computed by the following equations:

$$
D_{i, j, k}^{(l)}=\left\{\begin{array}{l}
D_{i, j, k}^{(1)}, \\
\text { if } i=\theta+1, j=C^{*}, k=0 ; \\
D_{i, j, k}^{(1)} \times\left(P_{i, j, k} D_{i-1, j, k}^{(l)}+Q_{i, j, k}\right), \\
\text { if } i \geq \theta+2, j=C^{*}, k=0 ; \\
D_{i, j, k}^{(1)} \times\left(P_{i, j, k} D_{i-1, j, k}^{(l)}+Q_{i, j, k} D_{i, j, k-1}^{(l-1)}\right), \\
\text { if } i \geq \theta+2, j=C^{*}, k>0 ; \\
D_{i, j, k}^{(1)} \times\left(P_{i, j, k} G_{i-1, j+1, k-1}^{(l-1)}+Q_{i, j, k} D_{i, j, k-1}^{(l-1)}\right), \\
\text { if } i=\theta+1, j=C^{*}, k>0 .
\end{array}\right.
$$

where

$$
D_{i, j, k}^{(1)}=\frac{i M_{m}+j M_{t}+k W_{t}}{i M_{m}+j M_{t}+(k+1) W_{t}}
$$

and

$$
\begin{aligned}
P_{i, j, k} & = \begin{cases}\frac{i M_{m}}{i M_{m}+j M_{t}+k W_{t}}, & \text { if } i \geq \theta+1, j=C^{*}, k>0 ; \\
0, & \text { otherwise. }\end{cases} \\
Q_{i, j, k} & = \begin{cases}\frac{j M_{t}+k W_{t}}{i M_{m}+j M_{t}+k W_{t}}, & \text { if } i \geq \theta+1, j=C^{*}, k>0 ; \\
0, & \text { otherwise. }\end{cases}
\end{aligned}
$$

Similar to the derivation for RRM, $\lambda_{h, t}$ and $P_{n c, t}$ for URM are expressed as that in (2) and (3), respectively, and the iterative approach applied to the analysis for RRM is used to obtain $\lambda_{h, t}, P_{o, t}, P_{f, t}$, and $P_{n c, t}$ for URM.

A new MBMS call is blocked when all dedicated logical channels are occupied, and the common logical channel is not turned on, and the system is at the $\mathbf{s}(i, j, k)$ state where $0 \leq$ $i \leq \theta, C-i \leq j \leq C, k \geq 0$ and $i \geq \theta+1, C^{*}+1 \leq j \leq C$, $k \geq 0$. The MBMS call blocking probability is

$$
P_{o, m}=\sum_{i=0}^{\theta} \sum_{j=C-i}^{C} \sum_{k \geq 0} \pi_{i, j, k}+\sum_{i \geq \theta+1} \sum_{j=C^{*}+1}^{C} \sum_{k \geq 0} \pi_{i, j, k} .
$$

The details of the derivation for the $P_{f, m}$ probability are given in pp. 8-9 [10], which is

$$
\begin{aligned}
P_{f, m} & =\sum_{i=0}^{\theta} \sum_{j=C-i}^{C} \sum_{k \geq 0}\left(1-W_{i, j, k}^{(k+i-C+j+1)}\right) \pi_{i, j, k} \\
& +\sum_{i \geq \theta+1} \sum_{j=C^{*}+1}^{C} \sum_{k \geq 0}\left(1-W_{i, j, k}^{(k+i-C+j+1)}\right) \pi_{i, j, k}
\end{aligned}
$$

where

$$
W_{i, j, k}^{(l)}=\left\{\begin{array}{cl}
W_{i, j, k}^{(1)}, \quad \text { if } 0 \leq i \leq \theta, j=C-i, k=0 \\
W_{i, j, k}^{(1)} \quad \times\left(L_{i, j, k} W_{i-1, j, k}^{(l-1)}+H_{i, j, k} W_{i, j, k-1}^{(l-1)}+\right. \\
& \left.M_{i, j, k} W_{i, j-1, k}^{(l-1)}+N_{i, j, k} W_{i-1, j+1, k-1}^{(l-1)}\right) \\
& \text { if } 0 \leq i \leq \theta, j=C-i, k>0 \\
& \text { or } 1 \leq i \leq \theta, C-i<j \leq C, k \geq 0 \\
& \text { or } i \geq \theta+1, C^{*}+1<j \leq C, k \geq 0 \\
& \times\left(L_{i, j, k} W_{i-1, j, k}^{(l-1)}+H_{i, j, k} W_{i, j, k-1}^{(l-1)}+\right. \\
W_{i, j, k}^{(1)} & \left.M_{i, j, k}+N_{i, j, k} W_{i-1, j+1, k-1}^{(l-1)}\right) \\
& \text { if } i \geq \theta+1, j=C^{*}+1, k \geq 0 .
\end{array}\right.
$$


and

$W_{i, j, k}^{(1)}=\frac{(C-j) M_{m}+j M_{t}+(i-C+j) W_{m}+k W_{t}}{(C-j) M_{m}+j M_{t}+(i-C+j+1) W_{m}+k W_{t}}$.

The derivations for $\lambda_{h, m}$ and $P_{n c, m}$ for URM are the same as that for RRM, which are

$$
\lambda_{h, m}=\frac{\eta \gamma\left(1-P_{o, m}\right) \lambda_{o, m}}{(\eta+\gamma) \mu_{m}+\eta \gamma P_{f, m}}
$$

and

$$
P_{n c, m}=P_{o, m}+\frac{\eta \gamma\left(1-P_{o, m}\right) P_{f, m}}{(\eta+\gamma) \mu_{m}+\eta \gamma P_{f, m}} .
$$

By using the iterative approach for the analysis of RRM, we obtain $\lambda_{h, m}, P_{o, m}, P_{f, m}$, and $P_{n c, m}$ for URM.

\section{Simulation Model}

This section describes the simulation models for the two channel allocation algorithms. Our models adopt the discrete event approach which has been widely used in many Personal Communications Service (PCS) studies [7], [11], [14]. In the models, we consider a $8 \times 8$ wrapped mesh cell structure. Denote Cell $(i, j)$ as one of wrapped mesh cells. A traditional user or an MBMS user resides in the non-overlapping area of a $\operatorname{Cell}(i, j)$ for a period $t_{x}$, then moves to the overlapping area of one of the four neighboring cells (selected with equal routing probabilities) for a period $t_{\tau}$, and finally moves to the non-overlapping area of one of the neighboring cells. The periods $t_{x}$ and $t_{\tau}$ are generated by the exponential or gamma random number generator. Each Cell $(i, j)$ provides a FIFO handoff queue to buffer the handoff calls due to the lack of free channels. Two counters, $C R_{t}(i, j)$ and $C R_{m}(i, j)$ are maintained for the $\operatorname{Cell}(i, j)$ to count the number of traditional calls and MBMS calls, respectively. The state, $S(i, j)$, indicates whether the $\operatorname{Cell}(i, j)$ turns on the common logical channel to serve the MBMS users.

We define six types of events as follows. The ARRIVAL event represents a new call arrival at a cell, which includes two fields: callType indicates whether the event is for a traditional call or an MBMS call; oldCell indicates the cell where the call is originated. The H_ARRIVAL event represents a softhandoff call arrival (i.e., a call in conversation moves into the overlapping area of the two cells). This event consists of six fields: callType is the same as that in the ARRIVAL event; $t_{r}$ stores the residual call holding time of the call; $t_{c}$ stores the elapsed connection time of a call before the event is processed; $t_{\tau}$ stores the residence time in the overlapping area; oldCell and newCell stores the ID of the cell which the user handoffs from and to, respectively. The H_COMPLETE event represents the completion of a soft-handoff call. The event consists of five fields, $t_{r}, t_{c}$, callType, oldCell and newCell, which are the same as those in the H_ARRIVAL event. The RELEASE event represents that the occupied channels are released at the old cell due to the forced termination of a handoff call or the completion of a handoff call. This event includes the callType and oldCell fields, which are the same as those in the H_ARRIVAL event. The COMPLETE event represents the completion of a call. This event consists of five fields. The $t_{c}$, callType, oldCell and newCell fields are the same as those in the H_ARRIVAL event. The area filed indicates that the call is completed in the non-overlapping area or overlapping area. The QDELETE event represents the deletion of an event from the handoff queue. The callType and newCell fields in this event are the same as those in the H_ARRIVAL event, and $t_{a}$ indicates the timestamp when a soft-handoff call is buffered in the handoff queue.

We maintain a timestamp, $t_{s}$, to indicate the time when an event occurs. The event are inserted into an event list and are deleted/processed from the list in a non-decreasing timestamp order. During the execution of simulation, a simulation clock, cur_ts, is maintained, which indicates the progress of the simulation. We calculate six output measures $P_{o, t}, P_{o, m}, P_{f, t}$, $P_{f, m}, P_{n c, t}$, and $P_{n c, m}$. Following the previous study [9], we use the Satisfaction Indication (denoted as $S I$ ) to reflect the customers' satisfaction about the network, which is defined as follows: Let $P_{c, t}\left(P_{c, m}\right)$ be the probability that a traditional (an MBMS) call is completed, $P_{i, t}\left(P_{i, m}\right)$ be the probability that a traditional (an MBMS) call is connected but is eventually force-terminated, $T_{i, t}\left(T_{i, m}\right)$ be the expected effective call holding time for a traditional (an MBMS) incomplete call, $T_{c, t}\left(T_{c, m}\right)$ be the expected effective call holding time for a traditional (an MBMS) complete call, and $E\left[T_{c, t}\right]=1 / \mu_{t}$ $\left(E\left[T_{c, m}\right]=1 / \mu_{m}\right)$ be the expected non-interrupted traditional (MBMS) call holding time. Then $S I$ is expressed as:

$$
\begin{aligned}
S I & =\left(\frac{\lambda_{o, t}}{\lambda_{o, t}+\lambda_{o, m}}\right)\left(\frac{P_{i, t} T_{i, t}+P_{c, t} T_{c, t}}{E\left[t_{c, t}\right]}\right) \\
& +\left(\frac{\lambda_{o, m}}{\lambda_{o, t}+\lambda_{o, m}}\right)\left(\frac{P_{i, m} T_{i, m}+P_{c, m} T_{c, m}}{E\left[t_{c, m}\right]}\right) .
\end{aligned}
$$

Note that since $P_{i, t} T_{i, t}+P_{c, t} T_{c, t} \leq \frac{1}{\mu_{t}}$ and $P_{i, m} T_{i, m}+$ $P_{c, m} T_{c, m} \leq \frac{1}{\mu_{m}}$, we have $0 \leq S I \leq 1$, and a larger $S I$ implies a higher customer satisfaction. The following variables are used to calculate output measures: the total number of ARRIVAL events $N_{t}\left(N_{m}\right)$ for traditional (MBMS) calls; the total number of handoff calls $N_{h, t}\left(N_{h, m}\right)$ for traditional (MBMS) calls; the total number of blocked traditional (MBMS) calls $N_{o, t}\left(N_{o, m}\right)$; the total number of traditional (MBMS) calls $N_{f, t}\left(N_{f, m}\right)$ that are forced to terminate; the total number of complete traditional (MBMS) calls $N_{c, t}$ $\left(N_{c, m}\right)$; the total effective call holding time $N T_{c, t}\left(N T_{c, m}\right)$ for complete traditional (MBMS) calls; the total effective call holding time $N T_{i, t}\left(N T_{i, m}\right)$ for incomplete traditional (MBMS) calls. We repeat the simulation runs until $N_{t}$ and $N_{m}$ exceed 5,000,000 to ensure the stability of the simulation results, and compute the output measures. Due to page limitation, we do not elaborate the models further.

\section{Simulation Validation}

The analytical models are validated by simulation experiments. In the implementation of the proposed analytical models, we use the $\mathrm{C}++$ programming language. We adopt the successive over-relaxation method to compute the steady state probabilities $\pi_{i}$ for RRM and $\pi_{i, j, k}$ for URM. As mentioned in [4], the complexity for the successive over-relaxation method is depend on the predefined threshold $\delta$ (used to obtain converge values of the steady state probabilities), and depend on the input parameter setups. Then we obtain the output 


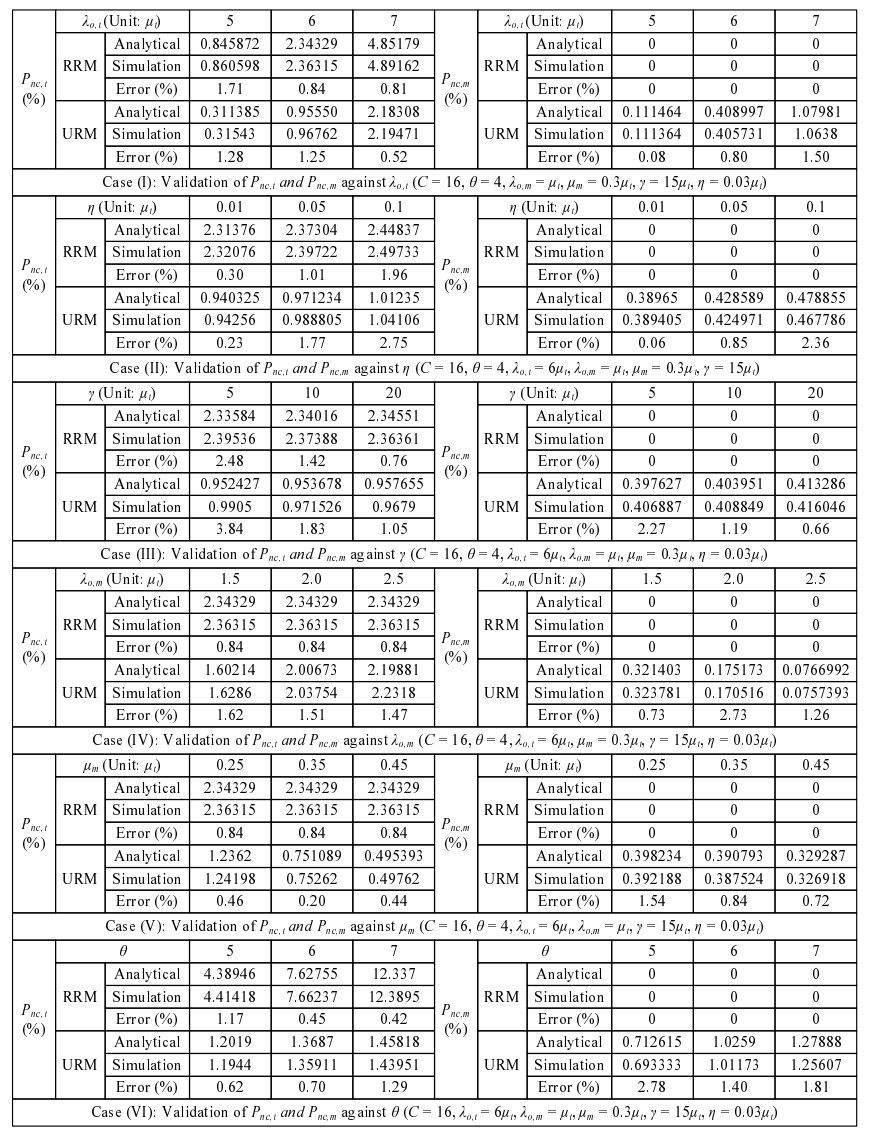

Fig. 4. Validation of simulation and analytic results

measures $\lambda_{h, t}, P_{o, t}, P_{f, t}, P_{n c, t}, \lambda_{h, m}, P_{o, m}, P_{f, m}, P_{n c, m}$ iteratively until the computation results converge to fixed values. The complexity of this iterative approach is dependent on the value of the predefined threshold $\delta$ and the input parameter setups. Most of operators used in the implementation of the analytical model is " + " and " $\times$ ", which are considered as the operators with low computation complexity.

Fig. 4 shows the $P_{n c, t}$ and $P_{n c, m}$ values of analytic models and simulation experiments for different $\lambda_{o, t}, \eta, \gamma, \lambda_{o, m}, \mu_{m}$, $\theta$ setups. In our study, the input parameters $\lambda_{o, t}, \lambda_{o, m}, \mu_{m}$, $\gamma$, and $\eta$ are normalized by $\mu_{t}$. For example, if the expected traditional call holding time is $1 / \mu_{t}=3$ mins, then $\lambda_{o, t}=6 \mu_{t}$ means that the expected time for two successive traditional call arrivals at a cell is 30 seconds. We assume that there are sixteen logical channels per cell, i.e., $C=16$. The figure shows the errors for $P_{n c, t}$ and $P_{n c, m}$ between simulation and analysis results for RRM and URM are within $3.84 \%$, and the analysis and the simulation results match well for different conditions.

\section{Performance Evaluation}

This section investigates the performance of RRM and URM. We investigate the effects of $\lambda_{o, t}, \lambda_{o, m}, \mu_{m}, \gamma, \eta$, and $\theta$ on the performance of RRM and URM. Then we investigate the effects of the variance of the UE residence time in the non-overlapping and overlapping areas. The parameter setups are given in the caption of each figure. Due to page limitation, in the following, we only describe the phenomena observed in

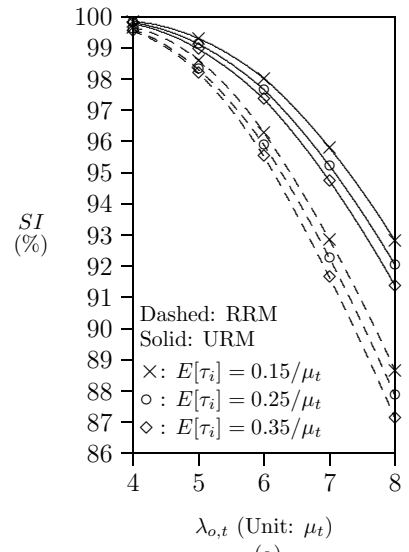

(a)

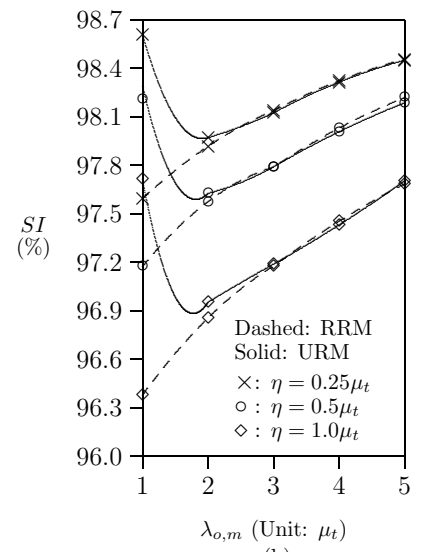

(b)
Fig. 5. (a) Effects of $\lambda_{o, t}$ and $E\left[\tau_{i}\right]\left(\theta=4, \lambda_{o, m}=\mu_{t}, \mu_{m}=0.25 \mu_{t}\right.$, $\left.\eta=0.5 \mu_{t}\right)$ (b) Effects of $\lambda_{o, m}\left(\theta=4, \lambda_{o, t}=6 \mu_{t}, \mu_{m}=0.25 \mu_{t}\right.$, $\left.\gamma=20 \mu_{t}\right)$

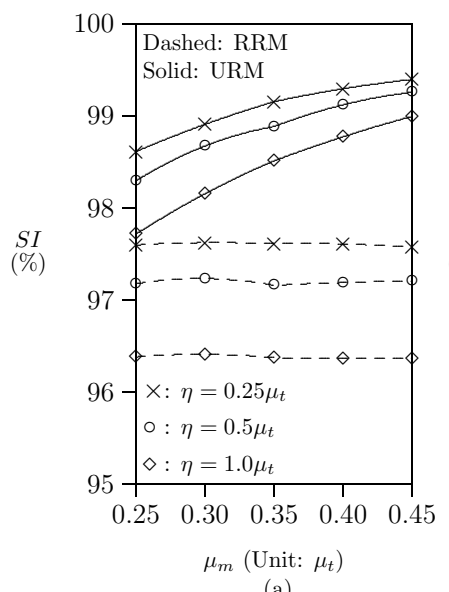

(a)

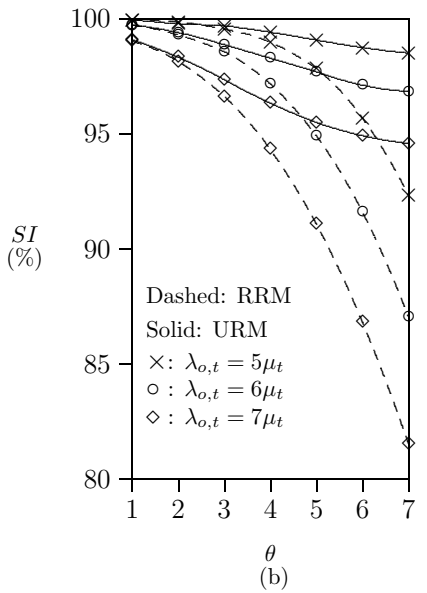

(b)
Fig. 6. (a) Effects of $\mu_{m}\left(\theta=4, \lambda_{o, t}=6 \mu_{t}, \lambda_{o, m}=\mu_{t}, \gamma=20 \mu_{t}\right)$ (b) Effects of $\theta\left(\lambda_{o, m}=\mu_{t}, \mu_{m}=0.25 \mu_{t}, \gamma=20 \mu_{t}, \eta=0.5 \mu_{t}\right)$

each figure. The reasons causing these phenomena has been treated as a technical report, and readers may refer [10].

Effects of $\lambda_{o, t}, \lambda_{o, m}, \mu_{m}$, and $\theta$ : Fig. 5 (a) plots $S I$ as a function of $\lambda_{o, t}$ with different $E\left[\tau_{i}\right]$ setups. We observe that $S I$ is a decreasing function of $\lambda_{o, t}$ for both URM and RRM. The URM outperforms RRM in terms of $S I$. Fig. 5 (b) studies the effects of $\lambda_{o, m}$. This figure indicates that when $\lambda_{o, m}$ increases (i.e., more MBMS call requests arrive in a period), the performance for URM approaches that for RRM. When $\lambda_{o, m}<3 \mu_{t}$, URM outperforms RRM in terms of SI. Fig. 6 (a) studies the effects of $\mu_{m}$. This figure indicates that for RRM, the effects of $\mu_{m}$ can be ignored. For URM, $S I$ increases when $\mu_{m}$ increases. Fig. 6 (b) investigates the effects of $\theta$ on the performance for RRM and URM. As shown in Fig. 6 (b), $S I$ is a decreasing function of $\theta$ for both URM and RRM.

Effects of $\tau_{i}$ and $x_{i}$ : Fig. 5 (a) also investigates the effects of the expected length of the overlapping period (i.e., $\left.E\left[\tau_{i}\right]\right)$ on the performance for URM and RRM. Fig. 5 (a) shows that with small $\lambda_{o, t}, E\left[\tau_{i}\right]$ has insignificant effects on $S I$ for URM and RRM, but with high $\lambda_{o, t}$, 


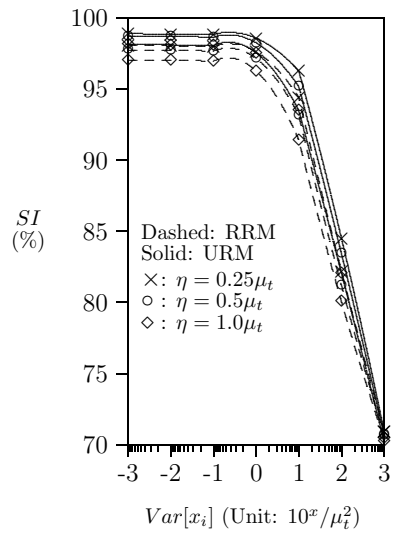

(a)

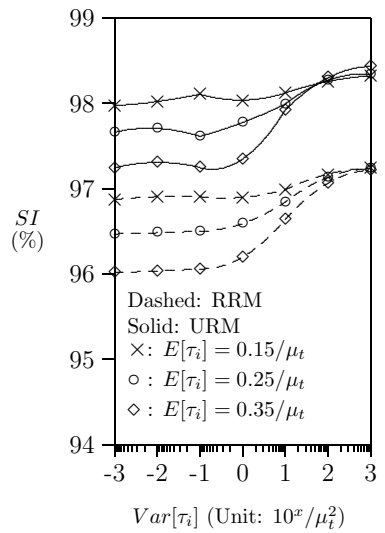

(b)

Fig. 7. (a) Effects of $\operatorname{Var}\left[x_{i}\right]\left(\theta=4, \lambda_{o, t}=6 \mu_{t}, \lambda_{o, m}=\mu_{t}, \mu_{m}=\right.$ $\left.0.25 \mu_{t}, \gamma=20 \mu_{t}\right)$ (b) Effects of $\operatorname{Var}\left[\tau_{i}\right]\left(\theta=4, \lambda_{o, t}=6 \mu_{t}, \lambda_{o, m}=\mu_{t}\right.$, $\left.\mu_{m}=0.25 \mu_{t}, \eta=0.5 \mu_{t}\right)$

as $E\left[\tau_{i}\right]$ increases, $S I$ for URM and RRM decreases. For effects of the non-overlapping period $E\left[x_{i}\right]=1 / \eta$, we observe the similar phenomena as those for the overlapping area. Due to page limitation, the performance figure for $E\left[x_{i}\right]=1 / \eta$ is ignored.

Effects of Variance of $\tau_{i}$ and $x_{i}$ : Based on the simulation experiments, we study the effects of variance of the nonoverlapping period and overlapping period, where we assume that the distributions for the non-overlapping period and overlapping periods are Gamma distributions with the variances $\operatorname{Var}\left[x_{i}\right]$ and $\operatorname{Var}\left[\tau_{i}\right]$, respectively. The Gamma distribution has been adopted in several PCS studies [9], [12], [13] to well simulate the UE cell residence time. Fig. 7 (a) shows that when $\operatorname{Var}\left[x_{i}\right] \leq \frac{1}{\mu_{t}^{2}}, S I$ for URM and RRM is insensitive to the change of the variance of the non-overlapping period. On the other hand, when $\operatorname{Var}\left[x_{i}\right]>\frac{1}{\mu_{+}^{2}}, S I$ degrades as $\operatorname{Var}\left[x_{i}\right]$ increases. Fig. 7 (b) studies effects of the variance of overlapping period, where we set $\lambda_{o, t}=6 \mu_{t}$ (high traditional call arrival rate) and $\lambda_{o, m}=\mu_{t}$ (low MBMS call arrival rate). Fig. 7 (b) shows that when $\operatorname{Var}\left[\tau_{i}\right] \leq \frac{1}{\mu_{t}^{2}}, S I$ for URM and RRM is insensitive to the change of the variance of the overlapping period. When $\operatorname{Var}\left[\tau_{i}\right]>\frac{1}{\mu_{t}^{2}}$, we observe $S I$ slightly increases.

\section{CONCLUSION}

This paper proposed analytic and simulation models to study the impact of MBMS service on the radio resource in the UMTS network. We considered two channel allocation algorithms, RRM and URM for the UMTS MBMS service. In RRM, a common logical channel is reserved to serve the MBMS calls. On the other hand, URM decides whether the dedicated logical channel or the common logical channel can be used to serve MBMS calls. In other words, if the number of MBMS calls in the system is larger than an interference threshold $\theta$, then URM turns on the common logical channel to serve the new incoming MBMS calls. In our study, to more precisely simulate the radio interface of UMTS, i.e., WCDMA, we apply the soft-handoff model for both traditional and MBMS calls while constructing analytic and simulation

models. Our performance evaluation results showed that URM outperforms RRM in terms of $S I$ (i.e., customer satisfaction indication) in all cases, and when the MBMS call arrival rate, $\lambda_{o, m}$, increases, the performance for URM approaches that for RRM.

\section{ACKNOWLEDGMENT}

The authors would like to thank the two anonymous reviewers. Their comments have significantly improved the quality of this paper.

\section{REFERENCES}

[1] 3GPP. 3rd Generation Partnership Project; Technical Specification Group Radio Access Network; Introduction of the Multimedia Broadcast/Multicast Service (MBMS) in the Radio Access Network (RAN); Stage 2 (Release 6). Technical Report 3G TS 25.346, 3GPP, Dec. 2004.

[2] 3GPP. 3rd Generation Partnership Project; Technical Specification Group Radio Access Network; Radio Resource Control (RRC); Protocol Specification (Release 6). Technical Report 3G TS 25.331, 3GPP, Mar. 2005.

[3] 3GPP. 3rd Generation Partnership Project; Technical Specification Group Radio Access Network; UTRAN functions, examples on signalling procedures (Release 6). Technical Report 3G TS 25.931, 3GPP, June 2005.

[4] G. Bolch, S. Greiner, H. Meer, and K. S. Trivedi, Queueing Networks and Markov Chains: Modeling and Performance Evaluation with Computer Science Applications. John Wiley and Sons Ltd, 1998.

[5] M. Chuah, T. Hu, and W. Luo, "UMTS release 99/4 airlink enhancement for supporting MBMS services," in Proc. IEEE International Conference on Communications, vol. 6, pp. 3231-3235, June 2004.

[6] P. J. Czerepinski, Y. M. Chapman, and J. Krause, "Coverage and planning aspects of MBMS in UTRAN," in Proc. Fifth IEE International Conference on 3G Mobile Communication Technologies, pages 529-533, 2004.

[7] Y. Fang, I. Chlamtac, and Y.-B. Lin, "Channel occupancy times and handoff rate for mobile computing and PCS networks," IEEE Trans. Computers, vol. 47, no. 6, pp. 679-692, 1998.

[8] H. Harri and T. Antti, WCDMA for UMTS. John Wiley and Sons Ltd, 2002.

[9] J.-Y. Jeng and Y.-B. Lin, "Equal resource sharing scheduling for PCS data services," ACM Wireless Networks, vol. 5, no. 1, pp. 41-55, January 1999.

[10] Y.-C. Lai, P. Lin, Y. Fang, and W.-H. Chen, Analytic models and performance evaluation for channel allocation for UMTS MBMS service, technical report, NTU, Dec. 2006.

[11] W. Li, Y. Fang, and R. R. Henry, "Actual call connection time characterization for wireless mobile networks under a general channel allocation scheme," IEEE Trans. Wireless Commun., vol. 1, no. 4, pp. 682-691, Oct. 2002.

[12] P. Lin, "Channel allocation for GPRS with buffering mechanisms," $A C M$ Wireless Networks, vol. 9, no. 5, pp. 431-441, Sept. 2003.

[13] P. Lin and Y.-B. Lin, "Channel allocation for GPRS," IEEE Trans. Veh. Technol., vol. 50, no. 2, pp. 375-387, Mar. 2001.

[14] Y.-B. Lin and A.-C. Pang, "Comparing soft and hard handoffs," IEEE Trans. Veh. Technol., vol. 49, no. 3, pp. 792-798, May 2000.

[15] K. Sipila, Z. Honkasalo, J. Laiho-Steffens, and A. Wacker, "Estimation of capacity and required transmission power of WCDMA downlink based on a downlink pole equation," in Proc. IEEE VTC2000-Spring, pp. 1003-1005, 2000.

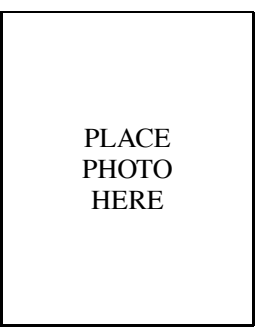

Yen-Cheng Lai (S'08) received the B.S. degree in computer science in 2002 from Tamkang University, Taipei County, Taiwan, and the M.S. and Ph.D. degrees in computer science and information engineering from National Taiwan University, Taipei, Taiwan, R.O.C., in 2004 and 2008, respectively. $\mathrm{He}$ is currently a Postdoctoral Researcher with the Institute of Applied Mechanics, National Taiwan University. His current research interests include design and analysis of PCS networks, wireless Internet and wireless PAN/LAN/WAN. 


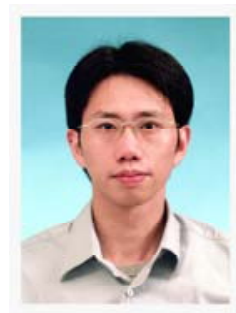

Phone Lin (M'02-SM'06) received his BSCSIE degree and Ph.D. degree from National Chiao Tung University, Taiwan, R.O.C. in 1996 and 2001, respectively. From August 2001 to July 2004, he was an Assistant Professor in Department of Computer Science and Information Engineering (CSIE), National Taiwan University, R.O.C. Since August 2004, he has been an Associate Professor in Department of CSIE and in Graduate Institute of Networking and Multimedia, National Taiwan University, R.O.C. His current research interests include personal communications services, wireless Internet, and performance modeling. Dr. Lin has published more than twenty international SCI journal papers (most of which are IEEE transactions and ACM papers). Dr. Lin is an Associate Editor for IEEE TRANSACTIONS ON Vehicular TeChNOLOGY, a Guest Editor for IEEE WIRELESS COMMUNICATIONS special issue on Mobility and Resource Management, and a Guest Editor for ACM/Springer MONET special issue on Wireless Broad Access. He is also an Associate Editorial Member for the WCMC Journal. Dr. Lin has received many research awards. He was elected as the Best Young Researcher, the 3rd IEEE ComSoc Asia-Pacific Young Researcher Award, 2007. He was a recipient of Research Award for Young Researchers from Pan Wen-Yuan Foundation in Taiwan in 2004, a recipient of K. T. Li Young Researcher Award honored by ACM Taipei Chapter in 2004, a recipient of Wu Ta You Memorial Award of National Science Council (NSC) in Taiwan in 2005, a recipient of Fu Suu-Nien Award of NTU in 2005 for his research achievements, and a recipient of 2006 Young Electrical Engineering Award, the Chinese Institute of Electrical Engineering. Dr. Lin is listed in Who's Who in Science and Engineering $(\mathrm{R})$ in 2006. Dr. Lin is a Senior Member, IEEE.

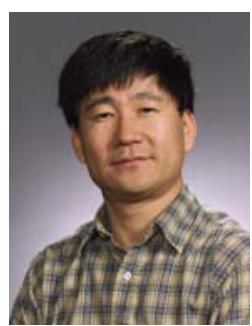

Yuguang “Michael” Fang (S'92-M'94-S'96-M'97SM'99-F'08) received a Ph.D. degree in Systems Engineering from Case Western Reserve University in January 1994 and a Ph.D degree in Electrical Engineering from Boston University in May 1997. He was an assistant professor in the Department of Electrical and Computer Engineering at New Jersey Institute of Technology from July 1998 to May 2000. $\mathrm{He}$ then joined the Department of Electrical and Computer Engineering at University of Florida in May 2000 as an assistant professor, got an early promotion to an associate professor with tenure in August 2003 and to a full professor in August 2005. He holds a University of Florida Research Foundation (UFRF) Professorship from 2006 to 2009. He has published over 200 papers in refereed professional journals and conferences. He received the National Science Foundation Faculty Early Career Award in 2001 and the Office of Naval Research Young Investigator Award in 2002. He is the recipient of the Best Paper Award in IEEE International Conference on Network Protocols (ICNP) in 2006 and the recipient of the IEEE TCGN Best Paper Award in the IEEE High-Speed Networks Symposium, IEEE Globecom in 2002. Dr. Fang is also active in professional activities. He is an Fellow of IEEE and a member of ACM. He has served on several editorial boards of technical journals including IEEE TRANSACTIONS ON COMMUNICATIONS, IEEE TRANSACTIONS ON WIRELESS COMMUNICATIONS, IEEE TRANSACTIONS on Mobile Computing and ACM Wireless Networks. He has been actively participating in professional conference organizations such as serving as the Steering Committee Co-Chair for QShine, the Technical Program ViceChair for IEEE INFOCOM'2005, Technical Program Symposium Co-Chair for IEEE Globecom'2004, and a member of Technical Program Committee for IEEE INFOCOM (1998, 2000, 2003-2008).

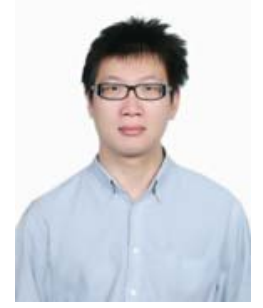

Wei-Hao Chen received his M.S. degree from $\mathrm{Na}-$ tional Taiwan University, Taipei, Taiwan, R.O.C., in 2005. His research interests include personal communications services, mobile IP, and wireless security. He currently is a senior engineer of HTC Corp. and develops several projects based on Windows Mobile PPC/SP. 